\title{
Lilo-Wawa festival: Lente sa pagsasalarawan ng pakikipag-ugnayang pantao (sarili at lipunan)
}

\author{
Galdo, Mark B. $\triangle$ \\ Southern Leyte State University - Tomas Oppus, Philippines (mgaldo05@gmail.com)
}

Morial, Donnabelle B.

Southern Leyte State University - Tomas Oppus, Philippines

De Paz, Michelle P.

Southern Leyte State University - Tomas Oppus, Philippines



ISSN: 2243-7703 Online ISSN: 2243-7711

OPEN ACCESS

Gozon, Manuel, Jr.

Southern Leyte State University - Tomas Oppus, Philippines

Pelesco, Maribel

Southern Leyte State University - Tomas Oppus, Philippines

Custillas, Fatima F.

Southern Leyte State University - Tomas Oppus, Philippines

\section{Abstract}

The Lilo-Wawa is a festival in the town of Liloan, Southern Leyte. It was formally launched in recognition of the beloved Patron Saint Ignatious Loyola - the founder of the Jesuits and patron of soldiers led by local officials and various public or private agencies. The purpose of this study is to describe the unique theme of human relations found at the Lilo-Wawa Festival in Liloan, Southern Leyte. Qualitative study was used in this study in a descriptive exploratory manner. A total of sixty (60) participants came from different groups (performers, conductors and dancers). Based on the results of the study, four themes of human interaction emerged. It is as follows: (1) cooperation and support of the people; (2) every action and movement shows an exchange of pleasure and joy; (3) courtesy; and (4) speech is not a hindrance to communion and harmony. The Lilo-Wawa festival has proven to be significant in human (self and social) relations in Liloan, Southern Leyte.

Keywords: festival, human relation, Lilo-Wawa, qualitative 


\section{Lilo-Wawa festival: Lente sa pagsasalarawan ng pakikipag-ugnayang pantao (sarili at lipunan)}

\section{Introduksyon}

Salaming pangkultural at pantradisyonal ng mga Pilipino ang festival. Nailalarawan nito ang pagkakaisa ng mga mamamayan sa lipunan maging ang mga karatig bayan nito. Ang festival ay hindi lamang eksibisyon ng mga sayaw, ngunit higit pa sa isang representasyon ng kultura, tradisyon, likas na yaman na maaring makita sa pakikipag-ugnayang pantao na nagsipaglahok, nakiisa at nakisama nito (Magpantay et al., 2014).

Patunay ito na ang festival ay isang abenyu ng pakikipag-ugnayang pantao na nagdudulot ng kahalagahan na nakatuon sa kolektibong interaksyon at pakikisama ng tao sa partikular na penomenang kinasasangkotan nito (Aguas, 2016). Kung kaya, gumagawa ang tao ng koneksyon at pakikipagsalamuha sa kapwa upang madama na kabilang o kasali sa pagdiriwang mapa-damdamin at pisikal man. Ang pagkakaroon ng mabuting ugnayan sa kapwa ay isa sa dalawang katangian ng kagandahang-loob na kung saan kakikitaan ito ng pagpapahalagang Pilipino na tinatawag na "linking social-personal value" o pagpapahalagang tulay ng sarili at lipunan na nagsisilbing kalinangan ng pag-unawa ng tao sa pagbabahagi ng karanasan (Yacat, 2013).

Sa pag-aaral, ang mga festival ay kadalasang nakatuon sa mga kultural na heograpiko sa lugar kung saan naitayo sa pamamagitan ng lipunan at ugnayan ng tao. Kaugnay pa rito, (Jaeger \& Mykletun, 2013) ang interaksyon ng tao sa kapwa ang nagpapakita ng matibay at pagkakaisa ng relasyon sa pagitan ng kapwa at ng festival. Sa lugar na pinangyarihan ng pakikipag-ugnayan, pinalagay na ang festival ay nakaambag sa pag-unlad ng pagkakakilanlan ng isang lipunan sa pamamagitan ng pagkukwento at pagkilala sa lipunan at kulturang kinabibilangan nito (Karlsen, 2007). Bagkus, ang pakikipag-ugnayan ay isang makabuluhang pakikisama ng isang tao tungo sa iba, sapagkat nagpapahiwatig ito ng makabuluhang palitan ng tunay na komunikasyon kahit sa panandalian lamang at mag-iiwan ng kasiyahan at kagalakan.

Ang mga festival ay bumubuo ng isang arena upang palakasin ang lokal na kultura, sining, at kasaysayan maging nag panlipunang etruktura kung saan naibahagi ang mga kultural na kasanayan at kamalayan, pati na rin ang paglikha ng mga lugar para sa panlipunang pakikiisa (Derrett, 2003).

Sa pagnanasang makatulong na mas makilala at mabigyang halaga ang kanilang pakikipag-ugnayan sa pamamagitan ng Lilo-Wawa festival ng mga taga-Liloan maging sa karatig lugar o sa Timogang Leyte. Upang sa gayon mapanatili at mapatibay ang kanilang pananalig sa kagandahan ng kanilang kultura at bukod sa lahat lalo pang maiintintidihan ng mga nakilahok at manonood kung ano ang tunay na kahulugan ng mga ginawang interaksyon o ipinakitang pakikipag-ugnayan sa pamamagitan ng Lilo-Wawa festival.

\section{Lilo-Wawa Festival at Kasaysayan Nito}

Ito ay isang religious na festival sa bayan ng Liloan, Southern Leyte. Kilala ito na Lilo-Lilo festival na kalauna'y binago at tinawag na Lilo-Wawa festival. Ang etimolohiya nito ay nagmula sa heograpikal na kinatatayuan ng bayan ng Liloan. Ang bayan ng Liloan ay isang "fourth class municipality" sa lalawigan ng Southern Leyte na paghati-hatian sa dalawampu't apat (24) na mga barangay. Ito ay matatagpuan sa hilagang bahagi ng isla ng Panaon. Ang islang ito ay konektado sa mainland sa pamamagitan ng isang tulay na tinawag na "wawa". Ang pangalang Liloan ay hango sa lokal na terminolohiyang "Lilo" na ang ibig sabihin ay puyo ng tubig o whirlpools. Kung kaya't, kilala na itong Lilo-Wawa festival (Choose Philippines, 2016).

Ang Lilo-Wawa festival ay pormal na inilunsad noong 2008 sa araw ng Hulyo 31 bilang pagkilala sa mahal na Patron Saint Ignatious Loyola - ang tagapagtatag ng Jesuits at patron ng mga sundalo. Ang festival na ito ay pinangunahan ng mga lokal na opisyales at ng iba't ibang ahensya mapa-publiko o pribado man. Kabilang dito 
Lilo-Wawa festival: Lente sa pagsasalarawan ng pakikipag-ugnayang pantao (sarili at lipunan)

ang pagpapakita ng pasasalamat sa mabuting ani, paggunita ng mga mahahalagang kaganapan sa lokal na kasaysayan tulad ng pagtanggap ng Kristiyanismo at pagdiriwang ng mga araw ng kapistahan ng mga santo. Sa Southern Leyte, ang pista ng Liloan ay nailalarawan sa pamamagitan ng umaapaw na pagkain, masayang musika at makulay na mga laro at paligsahan.

Ang premisong ito ay nagpahikayat sa mananaliksik na pag-aralan ang Lilo-Wawa festival sapagkat magiging tugon ito bilang lunsaran sa kontekstuwalisasyon at lokalisasyon sa pagtuturo-pagkatuto lalo na sa mag-aaral sa Southern Leyte. Isang instrumento din ito upang maipamalas at mapahalagahan ang natatanging kaugalian ng mga Lilo-anon o Southern Leyteno sa kabuuan. Kung kaya’t, makabuluhan ang pag-aaral na ito upang maitampok hindi lang ang makulay na selebrasyon ng Lilo-Wawa festival, kung hindi ang mga ugnayan ng selebrasyon sa tao na siyang nagbibigay-kulay at buhay sa selebrasyon. Nais patunayan ng pag-aaral na ito na ang festival ay isang lente sa pagsasalarawan ng pakikipag-ugnayang pantao. Mula sa mga pag-aaral, wala pang pag-aaral sa pakikipag-ugnayang pantao sa mga festival sa Pilipinas partikular sa Lilo-Wawa festival ng Liloan, Southern Leyte.

\subsection{Layunin ng Pag-aaral}

Layunin ng pag-aaral na ito ay mailarawan ang namumukod tanging temang nakapaloob sa pakikipag-ugnayang pantao na makikita sa pagdiriwang ng Lilo-Wawa Festival sa Liloan, Southern Leyte.

\section{Konseptwal na Balangkas ng Pag-aaral}

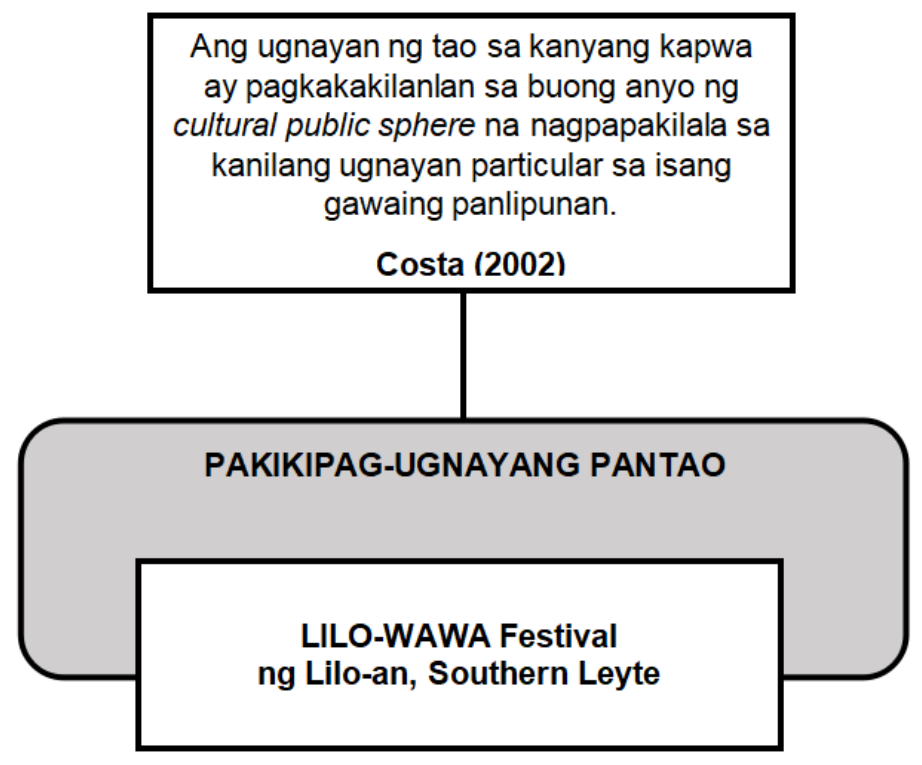

Pigura 1. Balangkas ng Pag-aaral

Ang pag-aaral na ito ay nakasalig sa ideya ni Costa (2002) na nagsasaad na ang ugnayan ng tao sa kanyang kapwa ay pagkakakilanlan sa buong anyo ng isang cultural public sphere na nagpapakilala sa ugnayang panlipunan. Patunay ito na sumasalamin sa ugnayang pantao ang kultura na napapalood sa isang lipunan. Ang kultura na ito ay natatangi lalong-lalo na sa pagdiriwang ng isang festival. Anupa't mas mailalahad ang karakter ng bawat tao sa isang lipunan kung papaano sila kumikilos at nagbibigay ng komplimento sa partikular na kasiyahan. Kung kaya, angkop ito sa pag-aaral sa Lilo-Wawa festival ng Liloan, Southern Leyte. 


\section{Metodolohiya}

Ginamit sa pag-aaral na ito ang kwalitatibong pag-aaral sa paraang descriptive exploratory. Ang pag-aaral na ito ay binigyang katugunan upang magkaroon ng isang malawak at matibay na kaalaman sa pagsusuri ng pakikipag-ugnayang pantao. Ang mga nakuhang datos ay masusing pinili ng mga mananaliksik mula sa mga napili nitong mga kalahok. Ang mga kalahok ay binubuo ng mga mamamayan na likas na nakatira sa Liloan, Southern Leyte na may edad 20 hanggang 40 taong gulang. Nagmula sa limang lugar na sakop ng Liloan (Poblacion, Himay-angan, Tabugon, San Roque at Duwang-an). May kabuuang anim-napung (60) kalahok na nagmula sa iba't ibang pangkat. Nagkaroon ng dalawang paraan sa pagkuha ng datos upang mailahad ang mahalagang datos gamit ang pakikipagpanayam sa mga tatlong grupo ng mga taong sangkot sa pag-aaral (awdyens, tagapamuno at mga mananayaw) at paggamit ng drone at video camera sa pagkuha ng mga mahahalagang kaganapan sa pagdiriwang na nagmula iba’t ibang domeyn.

\section{Resulta at Pagtatalakay}

Matutunghayan sa bahaging ito ang apat na kabuuang tema sa pakikipag-ugnayang pantao sa Lilo-Wawa festival ng Liloan, Southern Leyte na naging sandigan sa pagbibigay interpretasyon sa mga suliraning inilahad ng pananaliksik na ito. Makikita dito ang pagtatalakay sa apat na mahahalagang tema na binigyang pansin sa pag-aaral na isinagawa. Ang mga ito ay (1) kooperasyon at suporta ng mamamayan; (2) bawat aksyon at galaw ay nagpapakita ng pagpapalitan ng kasiyahan at kagalakan; (3) naipapalabas ang kagandahang-loob; at (4) hindi naging sagabal ang pananalita sa pakikipagkapwa at pagkakasundo.

\subsection{Kooperasyon at Suporta ng Mamamayan}

Sa pagdiriwang ng Lilo-Wawa festival, mas lumilitaw ang kooperasyon at pagkakaisa ng bawat mamamayan upang ipakita ang suporta nito sa selebrasyon. Makikita ang katangiang ito sa ugnayan ng mga taong naging bahagi ng kompetisyon sa sayawan at mga manonood. Ang ugnayang ito ay nagbibigay lakas at kumpiyansa sa mga nakilahok na ibigay ang husay at galing sa kanilang pagsasayaw. Ang madalas na pakikipag-ugnayan na sinasamahan ng kooperasyon na may dalang pagsuporta ay humihikayat ng positibong perspektiba kaugnay sa isang gawain (Hammit et al., 2006). Kung kaya sa pagpapahalagang Pilipino, ang bigkis ng isa't isa ay makikita na likas sa kinikilos ng mga tao. Magkakaroon ng malinaw at maayos na ugnayan ang bawat isa sa kadahilanang iisa ang tunguhin at ninanais upang magkaroon ng katagumpayan sa isinasagawang gawain.

Patunay na mahalaga ang suporta ng mamamayan sapagkat magkakaroon ng kulay at katagumpayan sa pagdiriwang. Bagkus, magiging maganda ang daloy $\mathrm{ng}$ isang pagdiriwang kung ang bawat isa ay nagkakaunawaan sa kung ano ang mga nakaatang na tungkulin upang mas maayos at malinaw ang selebrasyon. Kung kaya't sa panig ng mga mananayaw, nahihikayat at nagbibigay ito ng lakas na loob sa mga kalahok na kabilang sa selebrasyon na ipakita ang kanilang galing at maayos na pagprepresenta. Anila, may iba't ibang paraan ang mga mamamayan sa pagpapakita ng kanilang suporta sa selebrasyon.

Pinatunayan ito mula sa mga pahayag ng mga kalahok na nagpapakita ng malaking suporta lalong-lalo na sa pagdiriwang ng festival. Ito ay ang:

"Pagdala ug towel para trapuhan ang mga singot sa mga entrante ug pagpainum ug tubig.

Labina sa pagsyaget nga makaingun sila nga daghan ang mi suporta nila.” (P1)

"Pagtabang sa pagdala sa mga props ug gikinahanglan sa ilang pagsayaw" (P15)

“Aron ipakita ang suporta pinaagi sa pagsinggit, katawa ug pagpakpak.” (P4)

"Magdala ug placard ug di gani kartolina para makita ang suporta namo sa amo kauban" (P24)

36 Consortia Academia Publishing (A partner of Network of Professional Researchers and Educators) 
Lilo-Wawa festival: Lente sa pagsasalarawan ng pakikipag-ugnayang pantao (sarili at lipunan)

"Motan-aw mi ug masalmot sa mga kalingawan sa lungsod labina sa mga gihimong kalingawan sa munisipyo diin ang mga lumulopyo nagsuportahay sa usag-usag” (P11)

"As Local Government Unit ang amu ra financial assistance then dunay mga sakyanan para mukuha sa mga entrante sa ilang baryo.” (P6)

"Nagbahin mi ug igong pinansyal para aktibong mosalmot ug molihok ang mga taw sa festival"

Isang mabuting ugnayan ito sa iba't ibang pangkat ng tao sa lipunan. Ang ugnayan sa mga kasamahang mag-aaral sa kapwa mag-aaral ay ipinapakita sa pamamagitan ng pagdadala ng tubig para may maiinum ang mga kalahok habang nasa street dancing at tuwalya para pahirin ang pawis ng kapwa mag-aaral. Ang pagsuportang ito ay ipinapakita sa paraang pagkilos. Samantalang, sa ugnayan ng mga taong nanonood sa lumahok sa paligsahan sa sayawan ay ipinapakita sa pamamagitan ng pagsigaw at pagpalakpak. Ito ay sa paraang di-berbal na komunikasyon. Ang paraang ito ay nagbibigay sigla sa mga kalahok bilang nakikiisa sa ginagawang pagdiriwang kung saan mas lalong maipakita ang kanilang kumpiyansa sa sarili. Habang sa ugnayan ng pamahalaan at kalahok, ang suporta nito ay sa pamamagitan ng suportang pinansiyal. Dito ibinubuhos ng gobyerno ang kanilang pundo upang maging kaakit-akit at maganda ang kabuuang pagdiriwang ng festival. Dahil dito, magiging matagumpay ang naturang festival.

\subsection{Bawat aksyon at galaw ay nagpapakita ng pagpapalitan ng kasiyahan at kagalakan}

Nagpapakahulugan lamang ito na ang bawat aksyon na ipinapakita ay nagbibigay ng kasiyahan at kagalakan sa pagpapahayag ng tao sa kanyang sariling damdamin sa pamamagitan ng kumpas ng kamay, gawi at iba pa sa kasagsagan ng pagdiriwang. Ang bawat tao ay may kanya-kanyang aksyon na ipinapakita upang maipalabas ang kanyang sentimento o reaksyon sa kanyang nakita o nararamdaman. Sa pagdiriwang ng Lilo-Wawa, makikita ang iba't ibang estilo ng ng mga tao sa pagpapakita nito. Kagalakan ng mga tao ang maipahayag ang kanilang sariling damdamin sapagkat nasanayan na ng bawat isa na maging positibo sa pakikilahok sa festival.

Sa katunayan, makikita ang ugnayan ng tao sa kapwa kung may pagkakaisa at mabuting pakikitungo. Sa mga sitwasyong nangyayari sa kasagsagan ng festival, ang kahulugan na nakakabit sa mga lugar at ibinahagi ng mga komunidad ay nagsisilbing malakas na mapagkukunan ng pagkakakilanlan kung saan nagsisilbing ambag sa pagkakaisa at tunguhin nito (McCabe \& Stokoe, 2004).

Ang mga Pilipino partikular ang taga-Liloanon ay masiyahin at tunay na may pagmamahal sa pista. Kung kaya, palaging naghahanap ng dahilan upang maging masaya.

"Sa pakikipag-ugnayan sa kalahok at mga manonood kailangan palaging nakangiti para maipakita ang kasiglahan. Nararapat din na may epektibong communication sa mga kalahok para maayos ang presentation. May respesto at pagkilala sa kanya-kanyang katauhan.” (P8)

"Ang paraan ng pakikipag-ugnayan sa kapwa tao at maayos na pakikitungo basi sa pagtanghal." (P10)

"Ang ganitong paraan ay nagsisilbing pundasyon upang maging maganda ang relasyon $n g$ bawat isa. Sadyang likas nating mga pilipino ang makisalamuha at makipag ugnayan dahilan sa magandang kalooban na ating ipinapakita." (P13)

"Sa pamamagitan ng pagkakaroon ng magandang asal at mabuting pakikitungu sa mga tao at sa pagiging mabuting mananayaw at reaksyon sa mga mukha.'(P20)

Bawat aksyon na ipinapakita ay nagbibigay ng kasiyahan at kagalakan sa mga manonood ang pagpapakita ng magandang asal at mabuting pakikitungo ay siyang nagbibigay kasiyahan at kagalakan ng bawat isa na siyang ipinapakita ng mamayan sa kanilang lugar at nagbibigay kasiyahan sa mga turista ang ipinapakita nilang 
pakikitungo.

Ang kagalakan na maipakita ang damdamin ay nagsasaad na kung naipahayag natin ang ating mga saloobin ay naipakita natin ng tapat sa ating mga sarili na may sariling dignidad sa pagpapahayag ng sariling pagkaunawa sa mga bagay na nakapalibot sa lipunan. Ang isang indibidwal ay nakikibahagi sa iba pang mga tao sa kanyang kapaligiran ng mga ideya, halagahin, ng kung ano ang tama o mali at maging ng konsepto ng mga bagay o pangyayari (Wates, 2000).

\subsection{Naipapalabas ang Kagandahang- Loob}

Sa pagdiriwang ng festival, mas namamayani rin ang kagandahang loob bilang isa sa mga pagpapahalagang Pilipino na bumubuo sa balangkas ng pagkataong taga-Liloanon. Bawat tao ay mayroong pakiramdam na pagiging kaisa at may seguridad sa pamilya at lumalabas na ito'y isa sa mga prayoridad ng tao sa buhay, ito'y mabisa upang mas galingan pa sa mga bagay na pinagkakaabalahan sa susunod na pagkakataon at naipapakita sa partisipasyon ng mga tao sa mga bawat kaganapan sa lipunan (Magpantay et al. 2014).

“maraming aral ang makukuha mo sa panunuod manatili ang iyong pananampalaya kay Sr. St Ignatious. Magbigay ka ng importansya sa kultura ng Liloan.” (P43)

"Nakakatulong ang ganitong panonood gaya ng festival sapagkat itoy nagpapakita at nagbabahagi ng mga pinapahalagahang kultura sa kanilang lugar. Sa pamamagitan ng pagtatanghal ay mas lubusan na makilala ng mga turista ang kagandahan mayroon ang isang lugar" (P22)

Ang mabuting pakikitungo, pananampalataya at paggalang ay maituturing na biyaya na nagpapakita na ang mga Pilipino ay nagtataglay ng mga mabubuting katangian na kung saan ay ito ang kanilang ginagamit sa araw-araw na pakikisalamuha sa bawat isa.

Likas sa mga Pilipino ang pagkakaroon ng mga magagandang katangian na kung saan ay makikita ito sa ibat-ibang paraan ng kanilang pakikipag-ugnayan sa bawat isa. Kultura na ng mga Pilipino ang pagsunod sa mga kaugaliang natutunan o itinuro mula sa mga magulang, guro at mga ninuno at ito'y makikita sa paraan ng kanilang pakikitungo sa kanilang kapwa tulad na lang ng kanilang pamamaraan sa pakikipag-usap at pakikipaghalubilo sa mga taong nakapaligid sa kanila at ang kanilang patuloy sa paniniwala at pagpapahalaga sa mga santo o patron na kung saan ito ay kanilang sinasamba at pinupuri tuwing kapistahan at pinasasalamat sa lahat ng mga biyayang natatanggap.

Ito ay nagpapatunay na anumang uri ng estado sa buhay ay makikita ang pagkakaisa at pagkakaunawaan ng bawat indibidwal. Para sa mga Pilipinong napapagaan nito ang kanilang kalooban sa tuwing pumupunta sa Simbahan at nakikipista sa patron. Kapag ginagawa nila ito, sila ay nagkakaroon ng lakas na harapin ang buhay mula sa iba't ibang mga pagsubok.

\subsection{Hindi naging sagabal ang pananalita sa pakikipagkapwa at pagkakasundo}

Ang ibig sabihin nito ay hindi naging sagabal ang pananalita nang bawat isa upang magkakasundo at magkakaisa, hindi lingid sa ating kaalaman na may ibat-iba tayong baryasyon ng wika, pero hindi ito naging hadlang sa bawat tao na nakikisalamuha. Kahit iba't iba ang ating pananalita nanatili ang paggalang sa bawat isa. Sa kabila nito ay magkaroon ng mabuting pakikitungo sa mga taong may iba't ibang pananampalataya. Pinapahalagahan ng mga Pilipino ang pakikisama sa pamamagitan ng paggamit ng maggalang na pananalita upang maiwasan ang mga di-pagkakaunawaan sa ibang tao.

'Sa pakikipag-ugnayan sa kalahok at mga manonood kailangan palaging nakangiti para maipakita ang kasiglahan. Nararapat din na may epiktibong communication sa mga kalahok para maayos ang presentation. May respesto at pagkilala sa kanya-kanyang katauhan.” (P7)

38 Consortia Academia Publishing (A partner of Network of Professional Researchers and Educators) 
Lilo-Wawa festival: Lente sa pagsasalarawan ng pakikipag-ugnayang pantao (sarili at lipunan)

“Amo silang abi abihon, presensya ug pagtabang sa panglihok sa ilang mga gamit.” (P21)

“Encourage. Moral and emotional support. (P39)

"Ang aking paraan sa pakikipag-ugnayan ay mahinhin at maging friendly sa aking kausap para magiging mabait din siya sa akin.” (P6)

'Kami ay palakaibigan, kilala ang liloan o ang aming lugar na may mabuting puso, nakikipag-ugnayan kami sa pamagitan ng ngiti sa aming mga labi dahil ang pagkakaintindihan ay wala sa salita kundi sa puso at gawa ng iyong ipinapakita sa kapwa.” (P7)

Ang paraan ng pananalita ay nagbibigay respeto o pag uugali mayroon ang isang tao. Nararapat lamang na maging maingat tayo sa ating mga pananalita upang may magandang relasyon at magkakaunawaan ang bawat isa.

Ang mga salitang sagabal, pananalita, pakikipagkapwa at pagkakasundo ay lagi nating makikita sa pagdiriwang ng festival lalong-lalo na pakikisalamuha ng tao, ito'y nagbibigay lakas ng bawat indibidwal upang maging matagumpay ang mga isinasagawa na selebrasyon. Hindi naging sukatan ang pananalita upang magkaroon ng matagumpay na selebrasyon, nagsasalita ang tao para ibahagi o ipahayag ang kanyang nasasaloob, naiisip at nadarama.

\section{Konklusyon}

Ang Lilo-Wawa festival ng Liloan, Southern Leyte ay lente sa pagsasalarawan ng pakikipag-ugnayang pantao (sarili at lipunan). Patunay ito na ang bawat festival ay sumasagisag sa buhay at kultura ng isang lugar na siyang sumasalamin sa tao at sa lipunan. Ang pag-aaral na ito ay nagnanais na makatulong sa pamamagitan ng mga rekomendasyon. Batay sa mga natuklasan at nabuong kongklusyon: (1) magsagawa ng pag-aaral sa tunguhin at halaga ng festival sa paggamit ng berbal at di-berbal na komunikasyon; (2) magsagawa ang mga LGU o lokal na pamahalaan ng mga hakbangin upang mapreserba ang festival na ito sabay nang pag-preserba ng kanilang identidad bilang Liloanon ng Southern Leyte; at (3) gawing isang lunsaran ang pag-aaral na ito sa kontekstwalisasyon ng lalawigan para mas makilala ang pagdiriwang ito.

\section{Mga Sanggunian}

Aguas, J. J. (2016). The Filipino value of pakikipagkapwa-tao vis-à-vis Gabriel Marcel's notion of creative fidelity and disponibilite. Scientia, 5(2), 17-39.

Choose Philippines. (2016). Southern Leyte's Wawa bridge: A sight to behold, Leyte. https://www.choosephilippines.com/specials/festivals/4418/lilo-wawa-festival-liloan-southern-leyte

Costa, X. (2002). Festive traditions in modernity: The public sphere of the festival of the 'Fallas'in Valencia (Spain). The Sociological Review, 50(4), 482-504. https://doi.org/10.1177/003802610205000402

Derrett, R. (2003). Making sense of how festivals demonstrate a community's sense of place. Event Management, 8, 49-58. https://doi.org/10.3727/152599503108751694

Jaeger, K., \& Mykletun, R. J. (2013). Festivals, identities, and belonging. Event Management, 17(3), $213-226$. https://doi.org/10.3727/152599513x137088633777

Javier, Jr., R. E. (2010). Karanasan at konsepto ng panata ng kapistahan ng ilang pamilyang Pilipino sa Lucban, Quezon. Retrieved from https://www.sanbeda-alabang.edu.ph/bede/images/researchpublication/BedanJournalPsych/BJP2016v2143.pdf

Karlsen, S. (2007). The music festival as an arena for learning. Festspel in Pite Alvdal and matters of identity

[Unpublished Ph.D. thesis]. Luleå University of Technology, Luleå, Sweden.

Luna. (2015). Model of participation in a Filipino festival. Retrieve from https://Ipulaguna.edu.ph/wp-content/upload/20018/12/9-MODEL-OF-PARTICIPATION-IN-A-FILIPIN 
Galdo, M. B., Morial, D. B., De Paz, M. P., Gozon, M., Jr., Pelesco, M., \& Custillas, F. F.

O-FESTIVAL.pdf

Magpantay, J. H., Decena, K. J., de Torres, D. M., Garcia, M. J. K., Villalonos, A. S., Felicen, S. S., \& Dinglasan, K. (2014). Socio-cultural effects of festivals in Batangas Province, Philippines. Asia Pacific Journal of Education, Arts and Sciences, 1(4).

McCabe, S., \& Stokoe, E. H. (2004). Place and identity in tourists' accounts. Annals of Tourism Research, 31(3), 601-622. https://doi.org/10.1016/j.annals.2004.01.005

Wates. (2000). Model of participation in a Filipino festival. Retrieve from https://Ipulaguna.edu.ph/wp-content/upload/20018/12/9-MODEL-OF-PARTICIPATION-IN-A-FILIPIN O-FESTIVAL.pdf

Yacat, J. A. (2013). Tungo sa isang mas mapagbuong sikolohiya: Hamon sa makabagong Sikolohiyang Pilipino. DALUYAN: Journal ng Wikang Filipino, 19(2), 5-32.

Yodal, M., Cetinel, F., \& Uysal, M. (2006). An examination of festival motivation and perceived benefits relationship: Eskiehir International Festival. Journal of Convention and Event Tourism, 10(4), 276-291. https://doi.org/10.1080/15470140903372020 